\title{
A computational model coupling mechanics and electrophysiology in spinal cord injury
}

\author{
Antoine Jérusalem • Julián A. García-Grajales • \\ Angel Merchán-Pérez • José M. Peña
}

\begin{abstract}
Traumatic brain injury and spinal cord injury have recently been put under the spotlight as major causes of death and disability in the developed world. Despite the important ongoing experimental and modeling campaigns aimed at understanding the mechanics of tissue and cell damage typically observed in such events, the differentiated roles of strain, stress and their corresponding loading rates on the damage level itself remain unclear. More specifically, the direct relations between brain and spinal cord tissue or cell damage, and electrophysiological functions are still to be unraveled. Whereas mechanical modeling efforts are focusing mainly on stress distribution and mechanisticbased damage criteria, simulated function-based damage criteria are still missing. Here, we propose a new multiscale model of myelinated axon associating electrophysiological impairment to structural damage as a function of strain
\end{abstract}

and strain rate. This multiscale approach provides a new framework for damage evaluation directly relating neuron mechanics and electrophysiological properties, thus providing a link between mechanical trauma and subsequent functional deficits.

Keywords Computational model - Axon · Electrophysiology $\cdot$ Mechanics $\cdot$ Spinal cord injury

\section{Introduction}

Neuropathological and neurological traumatic brain injuries (TBIs) and spinal cord injuries (SCIs) ranging from simple edema or axonal injury to cognitive deficits or post-traumatic stress disorders have already been widely referenced for both animal models and humans (Hicks et al. 2010; Goldstein et al. 2012). At the cellular level, these injuries have been more specifically linked to cytoskeleton alterations, neurotransmitter deficits, disruption of calcium homeostasis, mitochondrial disturbances, loss in membrane permeability, demyelination and even altered gene expression (Cernak and Noble-Haeusslein 2010; Ouyang et al. 2010; Alford et al. 2011; Peter and Mofrad 2012). At the functional level, recent efforts by Shi and coworkers have quantified the injury level by measuring the membrane integrity and compound action potential (CAP) propagation under ex vivo electrical stimuli of guinea pig spinal cord white matter after tensile (Shi and Whitebone 2006), compressive (Ouyang et al. 2008) and blast loading (Connell et al. 2011).

Computational biomechanics simulations making use of finite element schemes have recently allowed for the identification of stress extrema and/or patterns at the tissue (Moore et al. 2009; Nyein et al. 2010; Cloots 2011; Cloots et al. 2013; Gupta and Przekwas 2013) and cell scales (Jérusalem 
and Dao 2012) during TBI events (see these references for a complete literature review). Conversely, recent work building on the observation of "leaky" voltage-gated sodium ion channel after trauma (Wang et al. 2009) has proposed a model of the resulting hyperpolarization-(left-)shifts of the ion channel current (Boucher et al. 2012). This model successfully reproduces the effect of the trauma-induced blebbing ${ }^{1}$ of a membrane patch on the electrophysiological properties of this patch. Building on the same left-shift mechanism, a similar study was proposed by Volman and $\mathrm{Ng}$ (2013), indicating a marked difference in the behavior of potentials in subthreshold ( $<14 \mathrm{mV}$ of potential shift) and supra-threshold $(>14 \mathrm{mV})$ traumas. Other approaches focusing on demyelination (a mark of multiple sclerosis) and its geometrical effect on the additional exposition of the membrane to the surrounding medium date back to the modeling work of Waxman and Brill (1978) which has been recently extended by Babbs and Shi (2013) to explore the effect of drug treatment on the conduction in the damaged region. Despite this growing interest in linking trauma and electrophysiological alterations, the intrinsic relation between mechanico-geometrical (stress, strain and strain rates) and electrophysiological alterations (potentials and currents) was left unmodeled in all these models, and a fully multiscale model of the fundamental link between the electrophysiological functions of the neuron-more specifically the propagation of action potentials (APs)—and its mechanical integrity is still lacking.

To this end, we have followed a three-step procedure: (i) we first developed a mechanical framework accounting for the multiscale deformation of the axonal components within the tissue and the resulting structural damage; (ii) as a second step, we developed a coupling model composed of two (geometrical and damage-based) components aimed at linking this mechanical framework to electrophysiological model parameters; and (iii) a one-dimension representative axon was discretized, in which the nodes of Ranvier (NRs) and the internodal regions (IRs) have been modeled using the Hodgkin-Huxley model and Cable Theory (Hodgkin and Huxley 1952; Koch 1999), respectively. In this approach, IRs are modeled as passive cables due to the multiple isolating myelin sheaths covering the axon membrane, while voltagegated ion channels responsible for the generation and propagation of the APs are concentrated at the NRs (Koch 1999). The approach is multiscale by nature, defining mechanical properties at the axon level, while linking the electrophysiological properties of the corresponding membrane in the NRs or IRs to the deformation and damage of the overall axon. As such, the intrinsically heterogeneous microscop-

\footnotetext{
${ }^{1}$ Axonal blebbing — or beading - consists of a series of undulating swellings at the NRs (Ochs et al. 1994; Markvin et al. 1999) leading to a loss of adhesion of the lipid bilayer to the underlying cortical axoplasm (Boucher et al. 2012).
}

ical mechanical and electrophysiological properties of the axonal components are homogenized at the mesoscale. Such approach, already widely used in the mechanical description of many types of materials [e.g., composite materials (Wu et al. 2013)] benefits from a lower computational cost and thus allow for simulations of coupled multiscale phenomena currently unavailable to mechanical models defined solely at the microscale.

Following calibration and validation against currently available experimental data, the proposed three-component model was finally shown to successfully predict the electrophysiological alterations ensuing a damaging mechanical event, while providing a new modeling tool able to extract data otherwise inaccessible through experimental means.

\section{Materials and methods}

The objective of coupling two mechanical and electrophysiological models into a single integrated model is to transfer the effects derived from an injuring mechanical cause into electrophysiological alterations affecting AP propagation, and ultimately cell behavior. The resulting model is presented in Fig. 1, where the mechanical, coupling (geometrical and damage-based) and electrophysiological models have been tagged as 1, 2 and 3, respectively. In the following, the building blocks of the overall model are described, followed by the finite difference scheme used to simulate the AP propagation impairment.

\subsection{Mechanical model}

Because of the complex association between the outer and inner faces of the axonal membrane (myelinated in the IRs) and its surrounding structures (extracellular matrix and cortical axoplasm), the microscopic fluid-like viscoelastic mechanical behavior of the membrane can be thought of as a fraction of the macroscopic mechanical behavior of the overall tissue. As such, and in view of the principal role of the membrane in the electrophysiology of the cell, a mechanical model focused around the membrane should be the first logical step. However, a much larger body of experimental data is available for the full axon mechanical and growth properties. Additionally, the intrinsic intertwined mechanical dependencies between membrane, cortical axoplasm and major cytoskeletal components such as microtubules naturally imply a quasi-one-to-one stretch and growth behavior between all these components (García et al. 2012). As a consequence, the passive and active behavior of the full axon is represented here by a Maxwell model (i.e., an elastic spring and a damper in series) and coupled to another viscous component representing the surrounding tissue, see Fig. 1-1. Note that other authors have also proposed an additional retracting 


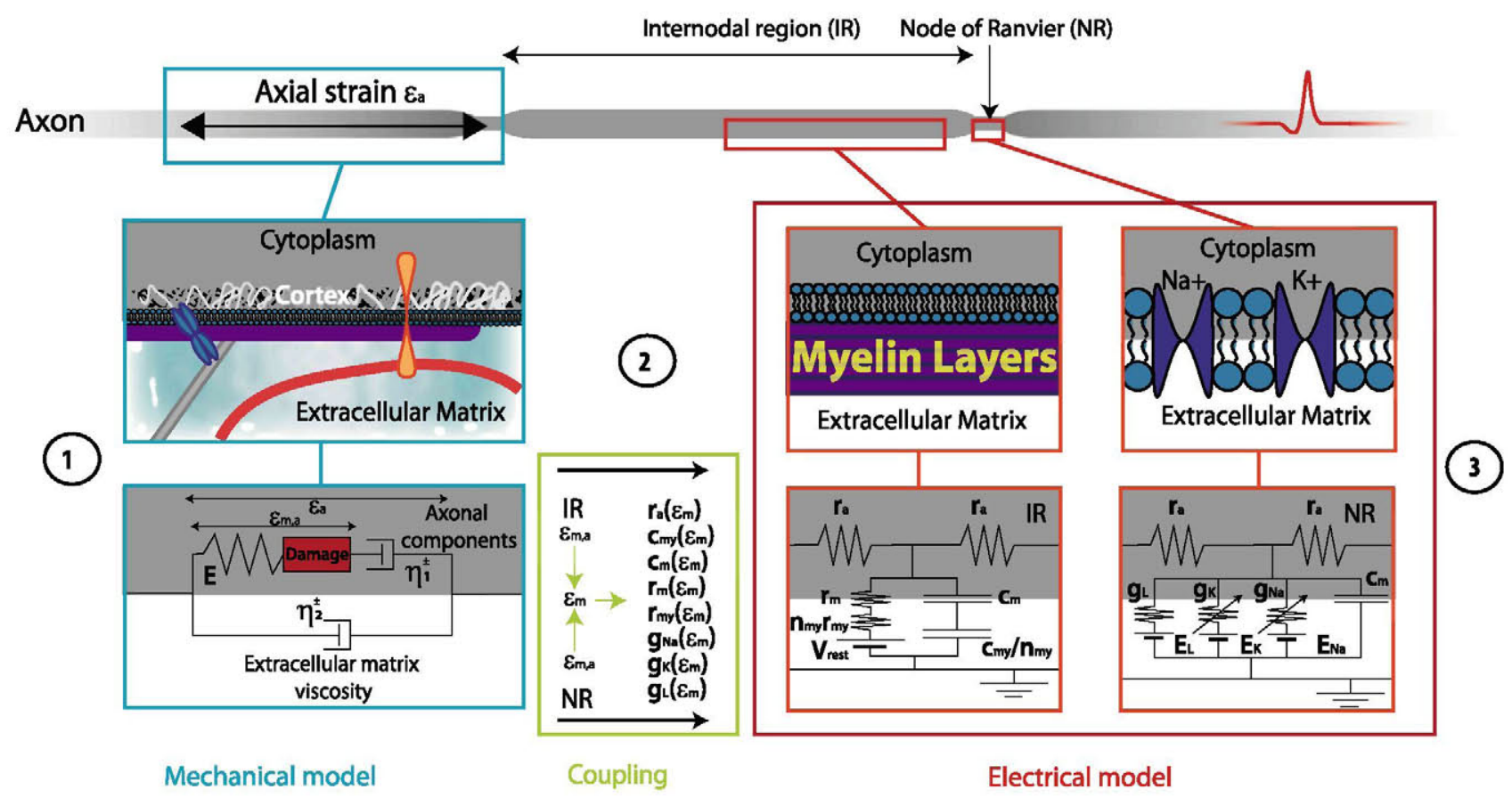

Fig. 1 Electrophysiological-mechanical framework: 1 mechanical model, 2 coupling model (geometrical and damage-based), 3 electrophysiological model

mechanism (Dennerll et al. 1989). We have decided to omit it in our model, as such effects are a priori not experimentally present at the rates under study.

This model implies the consideration of three parameters: $E, \eta_{1}^{ \pm}$and $\eta_{2}^{ \pm}$, respectively, the microscopic axial axonal stiffness (i.e., the spring in the Maxwell model), the tissue active molecular reorganization viscosity (i.e., the damper in the Maxwell model), and the viscous molecular crosslinking between the axon and its surrounding structures (i.e., the damper in parallel with the Maxwell model). More specifically, the viscous component of newtonian viscosity $\eta_{1}^{ \pm}$represents membrane turnover, (de)polymerization (e.g., microtubules) and (de)lipidation (e.g., the membrane and myelin sheaths), as well as any other active molecular reorganization driven by mechanical stretch or during relaxation (Dennerll et al. 1989). The superscript symbol " \pm " of $\eta_{1}^{ \pm}$and $\eta_{2}^{ \pm}$indicate that this value is a priori different under applied tension and during macroscopic stress-free relaxation. The microscopic axial axonal strain sustained by the molecular bonds of the axon (and thus membrane) is defined by $\varepsilon_{\mathrm{m}, \mathrm{a}}$ and the macroscopic overall axial strain by $\varepsilon_{\mathrm{a}}$.

A microscopic damage contribution with a corresponding strain $\varepsilon_{\mathrm{D}, \mathrm{a}}$ is finally considered so that the axonal stress

$\sigma_{1}=E\left(\varepsilon_{\mathrm{m}, \mathrm{a}}-\varepsilon_{\mathrm{D}, \mathrm{a}}\right)$

is only representative of the unbroken bonds. Inspired by metal plasticity yield evolution rules, an evolution equation of this irreversible damage strain is assumed to verify the following rule:
$\begin{cases}\dot{\varepsilon}_{\mathrm{D}, \mathrm{a}}=0, & \text { if } \sigma_{1}<\sigma_{0}+k \varepsilon_{\mathrm{D}, \mathrm{a}} \\ \sigma_{1}=\sigma_{0}+k \varepsilon_{\mathrm{D}, \mathrm{a}}, & \text { otherwise }\end{cases}$

where $\sigma_{0}$ is the damage stress threshold of the axon, and where $k$ is a model parameter representative of the damage evolution. This coupling between reversible-elastic and irreversible-plastic behaviors is justified by the observation that a relaxation of the membrane following large plastic deformation exhibits an elastic-reversible behavior upon reloading (Wang et al. 2009). Again, the deformation and failure of all axonal components are assumed to occur simultaneously.

Gathering Eqs. (1), (2), and the two newtonian components, the system of equation characterizing the full model reads:

$$
\left\{\begin{array}{l}
\sigma_{1}=E\left(\varepsilon_{\mathrm{m}, \mathrm{a}}-\varepsilon_{\mathrm{D}, \mathrm{a}}\right) \\
\sigma_{1}=\eta_{1}^{ \pm}\left(\dot{\varepsilon}_{\mathrm{a}}-\dot{\varepsilon}_{\mathrm{m}, \mathrm{a}}\right) \\
\sigma_{2}=\eta_{2}^{ \pm} \dot{\varepsilon}_{\mathrm{a}} \\
\sigma=\sigma_{1}+\sigma_{2} \\
\left(\sigma_{1}-\sigma_{0}-k \varepsilon_{\mathrm{D}, \mathrm{a}}\right) \dot{\varepsilon}_{\mathrm{D}, \mathrm{a}}=0 \text { and } \sigma_{1} \leq \sigma_{0}+k \varepsilon_{\mathrm{D}, \mathrm{a}}
\end{array}\right.
$$

It should be emphasized that the proposed model is the simplest mechanical model accounting for the experimentally observed loading and gradual unloading of the axon (along with the damage component). As a consequence, the elasticity of the surrounding extracellular matrix is neglected as a first approximation, thus avoiding an increase in the size of the parameter space. 
Additionally, despite large values of the macroscopic strain in the following simulations, the microscopic strain is less than $25 \%$ in the vast majority of the cases of this work, with larger values for the upcoming fast-severe cases and the fast-moderate case at $t=0 \mathrm{~min}$ (where the potential is anyway reduced to 0 ). As a consequence, the small strain assumption was considered acceptable as a first approximation (see also Sect. 4 below).

Finally, the equations are presented in a quasi-static framework, thus avoiding the need for additional dynamic terms. This choice is motivated by the observation that the electrophysiological measurements corresponding to the loading conditions used in the following do not exhibit any dynamic oscillations (Shi and Whitebone 2006), indicating that material deformation is not driven by its inertia, but by a slower viscous behavior.

\subsection{Geometrical coupling model}

The geometrical coupling model, see Fig. 1-2, first includes a strain model providing the NR and IR specific axial microscopic axonal strains, respectively, $\varepsilon_{\mathrm{m}, \mathrm{a}}^{\mathrm{IR}}$ and $\varepsilon_{\mathrm{m}, \mathrm{a}}^{\mathrm{NR}}$, based on their "average" counterpart $\varepsilon_{\mathrm{m}, \mathrm{a}}$ (i.e., the output of the mechanical model). As described in detail in Supplementary Material A, for a given structural composition of the white matter and a given average microscopic axial strain $\varepsilon_{\mathrm{m}, \mathrm{a}}$, we postulate the existence of a parameter $v \in[0,1]$ such that the microscopic axial strains of the NRs and IRs are given by

$$
\left\{\begin{array}{l}
\varepsilon_{\mathrm{m}, \mathrm{a}}^{\mathrm{NR}}=\frac{v}{\chi} \varepsilon_{\mathrm{m}, \mathrm{a}} \\
\varepsilon_{\mathrm{m}, \mathrm{a}}^{\mathrm{IR}}=\frac{1-v}{1-\chi} \varepsilon_{\mathrm{m}, \mathrm{a}}
\end{array}\right.
$$

where $\chi$ is the density of NRs per axonal unit length. Note that the three special cases $v=0, v=1$ and $v=\chi$ correspond, respectively, to: no strain in the NRs (the deformation is fully accommodated by the IRs), no strain in the IR (the deformation is fully accommodated by the NRs), and fully homogeneous strain in the whole axon. For simplicity, the notations will subsequently drop the "IR" and "NR" superscripts, and, unless indicated otherwise, all subsequent formulae apply to both regions (NR or IR) individually.

The second step of the coupling model links the microscopic surface membrane strain $\varepsilon_{\mathrm{m}}$ to the microscopic axial strain $\varepsilon_{\mathrm{m}, \mathrm{a}}$ by noting that, assuming axon incompressibility,

$1+\varepsilon_{\mathrm{m}}=\sqrt{1+\varepsilon_{\mathrm{m}, \mathrm{a}}}$

Under the same assumption, the diameter of an axon stretched macroscopically by $\varepsilon_{\mathrm{a}}$ is given by

$d=\frac{d_{0}}{\sqrt{1+\varepsilon_{\mathrm{m}, \mathrm{a}}}}$
The membrane thickness is additionally assumed to be constant based on the assumption that the "height" of the lipid bilayer remains the same during loading: $h=h_{0}$. In the previous equations, the subscripts " 0 " in $h_{0}$ and $d_{0}$ refer to their respective resting state values (no strain). The $n_{\text {my }}$ myelin layers in the IRs follow the same scaling laws for their own individual resting thickness $h_{\mathrm{my}, 0}$ and diameter $d_{\mathrm{my}, 0}^{i}=d_{0}+2 h_{0}+2(i-1) h_{\mathrm{my}, 0}$, where $i \in\left[1, n_{\mathrm{my}}\right]$ is the number of the layer.

As a final step, these relations correlate the microscopic mechanical viscoelastic stretch of the axonal regions IR and NR (characterized by $\varepsilon_{\mathrm{m}, \mathrm{a}}^{\mathrm{NR}}$ and $\varepsilon_{\mathrm{m}, \mathrm{a}}^{\mathrm{IR}}$ for the NRs and IRs, respectively) to their membrane surface-dependent electrical properties (characterized by the set of capacitances $c_{\mathrm{m}}, c_{\mathrm{my}}$, resistances $r_{\mathrm{a}}, r_{\mathrm{m}}$ and $r_{\mathrm{my}}$, and the leak, voltage-gated sodium and potassium channels $g_{\mathrm{L}}, g_{\mathrm{Na}}$ and $g_{\mathrm{K}}$ ); see Fig. 1-2 and Sect. 2.5 below. It is noticeable that these electrophysiological alterations are thus related to the change in membrane surface area, i.e., geometrically driven.

\subsection{Electrophysiological model}

The myelinated IR segments of the axon are simulated according to the Cable Theory as an electrical circuit involving the resting transmembrane potential $\left(V_{\text {rest }}\right)$, the axial resistivity of the axonal cytoplasm $\left(\rho_{\mathrm{a}}\right)$, the transmembrane resistivity $\left(\rho_{\mathrm{m}}\right)$, the resistivities of the myelin sheaths $\left(\rho_{\mathrm{my}}\right)$, and the cell membrane and myelin sheaths equivalent electric constants $\left(C_{\mathrm{m}}\right.$ and $\left.C_{\mathrm{my}}\right)$. Simulation of the NRs by the Hodgkin-Huxley model are based on the same parameters (except those pertaining to myelin and the membrane resistivity) plus the variable conductivities $\left(G_{\mathrm{Na}}\right.$ and $\left.G_{\mathrm{K}}\right)$ and potentials $\left(E_{\mathrm{Na}}\right.$ and $\left.E_{\mathrm{K}}\right)$ for the voltage-gated sodium $\left(\mathrm{Na}_{\mathrm{v}}\right)$ and potassium $\left(\mathrm{K}_{\mathrm{V}}\right)$ channels. Additionally, the membrane resistivity is replaced by a "leak" conductivity $\left(G_{\mathrm{L}}\right)$ accounting for the membrane resistivity along with other mechanisms related to the ion homeostasis (e.g., $\mathrm{Na}^{+} / \mathrm{Ca}^{2+}$ and $\mathrm{Na}^{+} / \mathrm{K}^{+}$exchangers). See Hodgkin and Huxley (1952) and Koch (1999) for more details.

Both Cable Theory and Hodgkin-Huxley models can be described by one unique partial differential equation (PDE):

$\mathrm{A} \frac{\partial^{2} V}{\partial x^{2}}=\mathrm{B} \frac{\partial V}{\partial t}+\mathrm{C} V+\mathrm{D}$

where $V$ is the membrane potential, and $\mathrm{A}, \mathrm{B}, \mathrm{C}$ and $\mathrm{D}$ are model parameters provided in Table 1 . In this table, $E_{\mathrm{L}}$ is chosen such that $V=V_{\text {rest }}$ at rest, i.e.,

$E_{\mathrm{L}}=\left(1+G_{\mathrm{Na}} / G_{\mathrm{L}}+G_{\mathrm{K}} / G_{\mathrm{L}}\right) V_{\text {rest }}-\left(G_{\mathrm{Na}} E_{\mathrm{Na}}+G_{\mathrm{K}} E_{\mathrm{K}}\right) / G_{\mathrm{L}}$ 
Table 1 PDE model parameters

\begin{tabular}{|c|c|c|}
\hline Parameter & Cable theory & Hodgkin-Huxley \\
\hline A & $\frac{\pi d_{0}^{2}}{4 \rho_{\mathrm{a}}}$ & $\frac{\pi d_{0}^{2}}{4 \rho_{\mathrm{a}}}$ \\
\hline B & $\left(\frac{h_{0}}{C_{\mathrm{m}} \pi d_{0}}+\sum_{i=1}^{n_{\mathrm{my}}} \frac{h_{\mathrm{my}, 0}}{C_{\mathrm{my}} \pi d_{\mathrm{my}, 0}^{i}}\right)^{-1}$ & $\frac{C_{\mathrm{m}} \pi d_{0}}{h_{0}}$ \\
\hline $\mathrm{C}$ & $\frac{\pi}{\frac{\rho_{\mathrm{m}} h_{0}}{d_{0}}+\sum_{i=1}^{n_{\mathrm{my}}} \frac{\rho_{\mathrm{my}} h_{\mathrm{my}, 0}}{d_{\mathrm{my}, 0}^{i}}}$ & $\frac{\pi d_{0}}{h_{0}}\left(G_{\mathrm{L}}+G_{\mathrm{Na}}+G_{\mathrm{K}}\right)$ \\
\hline $\mathrm{D}$ & $\frac{-\pi V_{\text {rest }}}{\frac{\rho_{\mathrm{m}} h_{0}}{d_{0}}+\sum_{i=1}^{n_{\mathrm{my}}} \frac{\rho_{\mathrm{myy}} h_{\mathrm{my}, 0}}{d_{\mathrm{my}, 0}^{i}}}$ & $\begin{array}{l}\frac{-\pi d_{0}}{h_{0}}\left(G_{\mathrm{L}} E_{\mathrm{L}}+\right. \\
\left.G_{\mathrm{Na}} E_{\mathrm{Na}}+G_{\mathrm{K}} E_{\mathrm{K}}\right)\end{array}$ \\
\hline
\end{tabular}

Table $2 \mathrm{Na}_{\mathrm{v}}$ and $\mathrm{K}_{\mathrm{v}}$ channel Hodgkin-Huxley evolution equations (potential and time units are, respectively, $\mathrm{mV}$ and $\mathrm{ms}$ in this table)

\begin{tabular}{ll}
\hline $\mathrm{Na}_{\mathrm{v}}$ & $\mathrm{K}_{\mathrm{v}}$ \\
\hline$G_{\mathrm{Na}}(V)=\bar{G}_{\mathrm{Na}} m^{3} h$ & $G_{\mathrm{K}}(V)=\bar{G}_{\mathrm{K}} n^{4}$ \\
$\frac{\mathrm{d} m}{\mathrm{~d} t}=\alpha_{\mathrm{m}}(V)(1-m)-\beta_{\mathrm{m}}(V) m$ & $\frac{\mathrm{d} n}{\mathrm{~d} t}=\alpha_{\mathrm{n}}(V)(1-n)-\beta_{\mathrm{n}}(V) n$ \\
$\frac{\mathrm{d} h}{\mathrm{~d} t}=\alpha_{\mathrm{h}}(V)(1-h)-\beta_{\mathrm{h}}(V) h$ & \\
$\alpha_{\mathrm{m}}(V)=\frac{25-\left(V-V_{\text {rest }}\right)}{10\left(e^{\left(25-\left(V-V_{\text {rest }}\right) / 10\right.}-1\right)}$ & $\alpha_{\mathrm{n}}(V)=\frac{10-\left(V-V_{\text {rest }}\right)}{100\left(e^{\left(10-\left(V-V_{\text {rest }}\right) / 10\right.}-1\right)}$ \\
$\alpha_{\mathrm{h}}(V)=0.07 e^{-\left(V-V_{\text {rest }}\right) / 20}$ & \\
$\beta_{\mathrm{m}}(V)=4 e^{-\left(V-V_{\text {rest }}\right) / 18}$ & $\beta_{\mathrm{n}}(V)=0.125 e^{-\left(V-V_{\text {rest }}\right) / 80}$ \\
$\beta_{\mathrm{h}}(V)=\frac{1}{e^{\left(30-\left(V-V_{\text {rest }}\right)\right) / 10}+1}$ & \\
\hline
\end{tabular}

In the special case of Hodgkin-Huxley, $G_{\mathrm{Na}}$ and $G_{\mathrm{K}}$ are not constant but depend on the time-dependent membrane potential $V$, and two constants $\bar{G}_{\mathrm{Na}}$ and $\bar{G}_{\mathrm{K}}$ (Hodgkin and Huxley 1952; Koch 1999), see Table 2. In this table, the corresponding evolution equations describe the probability of open versus closed states of gating particles $n, m$ and $h$. They need to be simultaneously opened in a given configuration ( $3 \mathrm{~m}$ 's and $1 h$ for $\mathrm{Na}_{\mathrm{v}} ; 4 \mathrm{~m}$ 's for $\mathrm{K}_{\mathrm{v}}$ ) to allow for the full opening of the gate, see Hodgkin and Huxley (1952) and Koch (1999) for further information.

\subsection{Damage coupling model}

The lack of protective myelin of the NRs (Shreiber et al. 2009), recent findings highlighting the primordial role of blast-damaged ion channels in neurobehavioral deficits (Park et al. 2013), and the $\mathrm{Na}_{\mathrm{v}}$ leak observed during trauma (Wang et al. 2009) suggest the need for a strain-based damage criterion specific to the ion channels' properties. This $\mathrm{Na}_{\mathrm{V}}$ (and in this model, $\mathrm{K}_{\mathrm{V}}$ ) leak has been rationalized by a hyperpolarizing shift (or left-shift) of transient current operational range (Wang et al. 2009) in the conductance of the ion channel. Boucher et al. (2012) proposed to capture this stretchinduced alteration by left-shifting the potential for $\mathrm{Na}_{\mathrm{v}}$ channels' activation and inactivation variables in the damaged part of the nodal axolemma. Despite the fact that such blebbing should a priori affect equally all types of ion channels, previous attempts to consider the leak of $\mathrm{K}_{\mathrm{V}}$ have not been fully pursued (Boucher et al. 2012). It has actually been suggested that the observed extracellular $\mathrm{K}^{+}$accumulation in post-traumatic rat hippocampal slices would be due to glial activity and not neuronal leak (D'A mbrosio et al. 1999). In the absence of further body of evidence, damage linked to blebbing or other type of damage are equally considered for both $\mathrm{K}_{\mathrm{v}}$ and $\mathrm{Na}_{\mathrm{v}}$ conductivity alterations.

The modeling approach of Boucher et al. (2012) involves the consideration of additional "left-shifted" $m$ and $h$ (see Table 2) calculated by increasing the potential $V$ in $\alpha_{\mathrm{m}}, \alpha_{\mathrm{h}}$, $\beta_{\mathrm{m}}$ and $\beta_{\mathrm{h}}$ by a value related to the degree of damage. These left shifted $m$ and $h$ are then weighed-averaged with their healthy counterparts to obtain the factor multiplying $\bar{G}_{\mathrm{Na}}$, see Table 2. We postulate here instead that both $\mathrm{Na}_{\mathrm{v}}$ and $\mathrm{K}_{\mathrm{v}}$ are being affected by the microscopic membrane strain, and that their corresponding reversal potentials $E_{\mathrm{Na}}$ and $E_{\mathrm{K}}$ should be tending toward 0 as the ion channels are increasingly leaking due to a loss of membrane integrity with increased damage (as dictated by the Nernst equation when ion concentrations on both sides of the membrane equilibrate themselves). Once a maximum value of microscopic membrane strain $\tilde{\varepsilon}$ is reached, the ion channel reversal potentials remain at 0 :

$$
\left\{\begin{array}{l}
E_{\mathrm{Na}}\left(\varepsilon_{\mathrm{m}}\right)= \begin{cases}E_{\mathrm{Na}, 0}\left(1-\left(\frac{\varepsilon_{\mathrm{m}}}{\tilde{\varepsilon}}\right)^{\gamma}\right), & \text { if } \varepsilon_{\mathrm{m}}<\tilde{\varepsilon} \\
0, & \text { else }\end{cases} \\
E_{\mathrm{K}}\left(\varepsilon_{\mathrm{m}}\right)= \begin{cases}E_{\mathrm{K}, 0}\left(1-\left(\frac{\varepsilon_{\mathrm{m}}}{\tilde{\varepsilon}}\right)^{\gamma}\right), & \text { if } \varepsilon_{\mathrm{m}}<\tilde{\varepsilon} \\
0, & \text { else }\end{cases}
\end{array}\right.
$$

where $E_{\mathrm{Na}, 0}$ and $E_{\mathrm{K}, 0}$ are the reference potentials of $\mathrm{Na}_{\mathrm{v}}$ and $\mathrm{K}_{\mathrm{V}}$, and where $\gamma$ is an exponent representing the sensitivity of the damage model to small versus large deformation.

As can be seen in Table 2, the parameters $\alpha_{\mathrm{m}}, \alpha_{\mathrm{n}}, \alpha_{\mathrm{h}}, \beta_{\mathrm{m}}$, $\beta_{\mathrm{n}}$ and $\beta_{\mathrm{h}}$ are only functions of $V$. However, because these parameters are intrinsically related to the evolution of the ion channel potential, the accompanying parameters originally calibrated by Hodgkin and Huxley (1952) can a priori be considered to implicitly include the contribution of the corresponding reversal potentials $E_{\mathrm{Na}}$ and $E_{\mathrm{K}}$, so that the parameters $\alpha_{\mathrm{m}}, \alpha_{\mathrm{h}}, \beta_{\mathrm{m}}$ and $\beta_{\mathrm{h}}$ are actually functions of $V-E_{\mathrm{Na}}$, and $\alpha_{\mathrm{n}}$ and $\beta_{\mathrm{n}}$, of $V-E_{\mathrm{K}}$, i.e., the difference between the membrane potential and the corresponding reversal potentials. As a consequence, an increase or decrease in the reversal potentials of $\mathrm{Na}_{\mathrm{v}}$ or $\mathrm{K}_{\mathrm{v}}$ by a given value as proposed by Eq. (9) should be translated into a modification by the contrary of this value in the potentials of parameters $\alpha_{\mathrm{m}}, \alpha_{\mathrm{n}}, \alpha_{\mathrm{h}}, \beta_{\mathrm{m}}, \beta_{\mathrm{n}}$ and $\beta_{\mathrm{h}}$. For a surface membrane strain $\varepsilon_{\mathrm{m}}$, these parameters are thus given by:

$$
\begin{aligned}
& \alpha_{\mathrm{m}}\left(V, \varepsilon_{\mathrm{m}}\right)= \begin{cases}\alpha_{\mathrm{m}}\left(V+\left(\frac{\varepsilon_{\mathrm{m}}}{\tilde{\varepsilon}}\right)^{\gamma} E_{\mathrm{Na}, 0}\right), & \text { if } \varepsilon_{\mathrm{m}}<\tilde{\varepsilon} \\
\alpha_{\mathrm{m}}\left(V+E_{\mathrm{Na}, 0}\right), & \text { else }\end{cases} \\
& \alpha_{\mathrm{h}}\left(V, \varepsilon_{\mathrm{m}}\right)= \begin{cases}\alpha_{\mathrm{h}}\left(V+\left(\frac{\varepsilon_{\mathrm{m}}}{\tilde{\varepsilon}}\right)^{\gamma} E_{\mathrm{Na}, 0}\right), & \text { if } \varepsilon_{\mathrm{m}}<\tilde{\varepsilon} \\
\alpha_{\mathrm{h}}\left(V+E_{\mathrm{Na}, 0}\right), & \text { else }\end{cases}
\end{aligned}
$$




$$
\begin{aligned}
& \beta_{\mathrm{m}}\left(V, \varepsilon_{\mathrm{m}}\right)= \begin{cases}\beta_{\mathrm{m}}\left(V+\left(\frac{\varepsilon_{\mathrm{m}}}{\tilde{\varepsilon}}\right)^{\gamma} E_{\mathrm{Na}, 0}\right), & \text { if } \varepsilon_{\mathrm{m}}<\tilde{\varepsilon} \\
\beta_{\mathrm{m}}\left(V+E_{\mathrm{Na}, 0}\right), & \text { else }\end{cases} \\
& \beta_{\mathrm{h}}\left(V, \varepsilon_{\mathrm{m}}\right)= \begin{cases}\beta_{\mathrm{h}}\left(V+\left(\frac{\varepsilon_{\mathrm{m}}}{\tilde{\varepsilon}}\right)^{\gamma} E_{\mathrm{Na}, 0}\right), & \text { if } \varepsilon_{\mathrm{m}}<\tilde{\varepsilon} \\
\beta_{\mathrm{h}}\left(V+E_{\mathrm{Na}, 0}\right), & \text { else }\end{cases} \\
& \alpha_{\mathrm{n}}\left(V, \varepsilon_{\mathrm{m}}\right)= \begin{cases}\alpha_{\mathrm{n}}\left(V+\left(\frac{\varepsilon_{\mathrm{m}}}{\tilde{\varepsilon}}\right)^{\gamma} E_{\mathrm{K}, 0}\right), & \text { if } \varepsilon_{\mathrm{m}}<\tilde{\varepsilon} \\
\alpha_{\mathrm{n}}\left(V+E_{\mathrm{K}, 0}\right), & \text { else }\end{cases} \\
& \beta_{\mathrm{n}}\left(V, \varepsilon_{\mathrm{m}}\right)= \begin{cases}\beta_{\mathrm{n}}\left(V+\left(\frac{\varepsilon_{\mathrm{m}}}{\tilde{\varepsilon}}\right)^{\gamma} E_{\mathrm{K}, 0}\right), & \text { if } \varepsilon_{\mathrm{m}}<\tilde{\varepsilon} \\
\beta_{\mathrm{n}}\left(V+E_{\mathrm{K}, 0}\right), & \text { else }\end{cases}
\end{aligned}
$$

Note that in the case of $\mathrm{Na}_{\mathrm{v}}$, as $E_{\mathrm{Na}}>0$, this approach is in agreement with the one proposed by Boucher et al. (2012) as damage will decrease $E_{\mathrm{Na}}$ through Eq. (9) and thus increase $V-E_{\mathrm{Na}}$ through Eq. (10).

Finally, these mechanical alterations automatically should a priori imply modifications of $E_{\mathrm{L}}$. Three possibilities were studied. The first choice assumed that the ion homeostasis exchangers would remain insensitive to all alterations and that $E_{\mathrm{L}}$ would thus remain constant. The second choice assumed that the ion homeostasis exchangers would not be damaged during deformation but would try to accommodate the changes in concentrations due to alterations of $\mathrm{Na}_{\mathrm{V}}$ and $\mathrm{K}_{\mathrm{V}}$ by still following Eq. (8), where conductances and reversal potentials are modified following Eqs. (9) and (10). The third choice assumed the latter change with Eq. (8), but also an additional modification of $E_{\mathrm{L}}$ following a similar expression as for $E_{\mathrm{Na}}$ and $E_{\mathrm{K}}$ in Eq. (9). The first assumption led to strong oscillations insensitive to any input currents, a phenomenon which was not observed experimentally and was thus discarded. The third case naturally implies the additional identification of $E_{\mathrm{L}}$-specific $\tilde{\varepsilon}$ and $\gamma$ (which are, a priori, different from their $\mathrm{Na}_{\mathrm{V}}$ and $\mathrm{K}_{\mathrm{V}}$ counterparts). Such identification is far from being straightforward as the signal was found to be weakly sensitive to $E_{\mathrm{L}}$ for small and large values of the microscopic strain, while switching abruptly to spontaneous firings in-between where the main triggering signals dies out quickly for moderate strain. As a consequence, the second assumption was chosen.

\subsection{Finite difference discretization of the final model}

Our in-house dedicated finite difference software Neurite was used for the discretization and calculations of Eq. (7). The approach consists of a 1D finite difference scheme discretizing the succession of myelinated IRs and NRs forming one cylindrical representative axon, see Fig. 1-3. Temporal and spatial convergences were systematically verified for all the simulations presented in this work.

The following relations are derived by considering the resistances, capacitances and conductances of unit deformed elements [characterized by Eqs. (5) and (6)] of given resistivities, capacities and conductivities.
For a given stretched spatial discretization step $\Delta x^{\mathbb{R} / N R}=$ $\Delta x_{0}\left(1+\varepsilon_{\mathrm{m}, \mathrm{a}}^{\mathbb{R} / \mathrm{NR}}\right)^{2}$ and an IR/NR-specific microscopic axial strain $\varepsilon_{\mathrm{m}, \mathrm{a}}^{\mathrm{RR} / \mathrm{NR}}$ (superscripts are dropped subsequently for clarity), the axoplasm, membrane, myelin layer $i$ and myelinated membrane element resistances are thus given for each region by

$$
\left\{\begin{array}{l}
r_{\mathrm{a}}=\frac{4 \rho_{\mathrm{a}}\left(1+\varepsilon_{\mathrm{m}, \mathrm{a}}\right)}{\pi d_{0}^{2}} \Delta x \\
r_{\mathrm{m}}=\frac{\rho_{\mathrm{m}} h_{0} \sqrt{1+\varepsilon_{\mathrm{m}, \mathrm{a}}}}{\pi d_{0} \Delta x} \\
r_{\mathrm{my}}^{i}=\frac{\rho_{\mathrm{m}} h_{\mathrm{my}, 0} \sqrt{1+\varepsilon_{\mathrm{m}, \mathrm{a}}}}{\pi d_{\mathrm{my}, 0}^{i} \Delta x} \\
r_{\mathrm{mm}}=r_{\mathrm{m}}+\sum_{i=1}^{n_{\mathrm{my}}} r_{\mathrm{my}}^{i}
\end{array}\right.
$$

where $n_{\mathrm{my}}=0$ for all NRs, and where use was made of Eqs. (5) and (6). Again, it must be emphasized that here and subsequently, $\Delta x$ is the stretched discretization step.

Similarly, the individual membrane, myelin layer $i$ and myelinated membrane element capacitances are given by

$$
\left\{\begin{array}{l}
c_{\mathrm{m}}=\frac{C_{\mathrm{m}} \pi d_{0}}{h_{0} \sqrt{1+\varepsilon_{\mathrm{m}, \mathrm{a}}}} \Delta x \\
c_{\mathrm{my}}^{i}=\frac{C_{\mathrm{my}} \pi d_{\mathrm{my}, 0}^{i}}{h_{m y, 0} \sqrt{1+\varepsilon_{\mathrm{m}, \mathrm{a}}}} \Delta x \\
c_{\mathrm{mm}}=\left(1 / c_{\mathrm{m}}+\sum_{i=1}^{n_{\mathrm{my}}} 1 / c_{\mathrm{my}}^{i}\right)^{-1}
\end{array}\right.
$$

Finally, the Hodgkin-Huxley leak and voltage dependent element conductances are given by

$$
\left\{\begin{array}{l}
g_{\mathrm{L}}=\frac{\pi d_{0} G_{\mathrm{L}}}{h_{0} \sqrt{1+\varepsilon_{\mathrm{m}, \mathrm{a}}}} \Delta x \\
g_{\mathrm{Na}}(V)=\frac{\pi d_{0} G_{\mathrm{Na}}\left(V, \varepsilon_{\mathrm{m}}\right)}{h_{0}\left(1+\varepsilon_{\mathrm{m}, \mathrm{a}}\right)} \Delta x \\
g_{\mathrm{K}}(V)=\frac{\pi d_{0} G_{\mathrm{K}}\left(V, \varepsilon_{\mathrm{m}}\right)}{h_{0}\left(\mathbf{1}+\varepsilon_{\mathrm{m}, \mathrm{a}}\right)} \Delta x
\end{array}\right.
$$

where $G_{\mathrm{Na}}\left(V, \varepsilon_{\mathrm{m}}\right)$ and $G_{\mathrm{Na}}\left(V, \varepsilon_{\mathrm{m}}\right)$ are the voltage and strain-dependent electrical conductivities of the $\mathrm{Na}_{\mathrm{V}}$ and $\mathrm{K}_{\mathrm{V}}$ channels. Finally, under the assumption that the finite number of ion channels (albeit damaged) remains constant during deformation, the strain dependency of both electrical conductances $g_{\mathrm{Na}}$ and $g_{\mathrm{K}}$ should only arise from the strain dependency of their conductivity counterparts $G_{\mathrm{Na}}$ and $G_{\mathrm{K}}$. This is ensured by "normalizing" the membrane surface deformed area $\pi d \Delta x$ by its surface stretch $1+\varepsilon_{\mathrm{m}}$, making use of Eq. (5) and recalling that $\Delta x=\Delta x_{0}\left(1+\varepsilon_{\mathrm{m}, \mathrm{a}}\right)$, thus leading to the strain-independence of $\frac{\pi d_{0} \Delta x}{h_{0}\left(1+\varepsilon_{\mathrm{m}, \mathrm{a}}\right)}$. The leak conductance $g_{\mathrm{L}}$ is on the other hand left strain-dependent. This choice is motivated by the fact that the membrane resistivity in Eq.

\footnotetext{
${ }^{2}$ In the Lagrangian framework followed by Neurite, $\Delta x=\Delta x_{0}(1+$ $\left.\varepsilon_{\mathrm{m}, \mathrm{a}}\right)$ where $\Delta x_{0}$ is the spatial discretization step for $\varepsilon_{\mathrm{m}, \mathrm{a}}=0 ; \Delta x$ is then automatically refined by Neurite when needed, to ensure spatial convergence.
} 
(11) is also strain-dependent, and that the activity of the ion homeostasis exchangers are assumed to be proportional to the membrane area as a first approximation.

\section{Results}

In the following, the analytical derivation of Eq. (3) is first analyzed in terms of irreversible damage evolution. A calibration is then proposed based on the experimental work of Shi and Whitebone (2006).

\subsection{Damage analysis}

The post-loading strain relaxation equation proposed in the following is derived from the system of equations (3). For this section only, we assume axial axonal loadings at constant strain rate $\dot{\varepsilon}_{0}$ until a maximum applied strain $\varepsilon_{\max }$ is reached; the unloadings are then accomplished free of stress. For a given combination of strain and strain rate, two cases are thus possible: in the first one, the combination of strain and strain rate does not irreversibly damage the axon, whereas in the second one, damage is accumulated following the evolution equations below. Figure 2 shows a representation of both regions in a $\dot{\varepsilon}_{0}$ versus $\varepsilon_{\max }$ plot using the parameters calibrated in the next section.

Setting the time origin when $\varepsilon_{\max }$ is reached, i.e., at the onset of unloading, the analytical integration of the system of equations (3) yields

$\varepsilon_{\mathrm{m}, \mathrm{a}}(t)=\left(\varepsilon_{\mathrm{m}, \mathrm{a}}^{*}-\varepsilon_{\mathrm{D}, \mathrm{a}}^{*}\right) e^{-t / \tau^{-}}+\varepsilon_{\mathrm{D}, \mathrm{a}}^{*}$

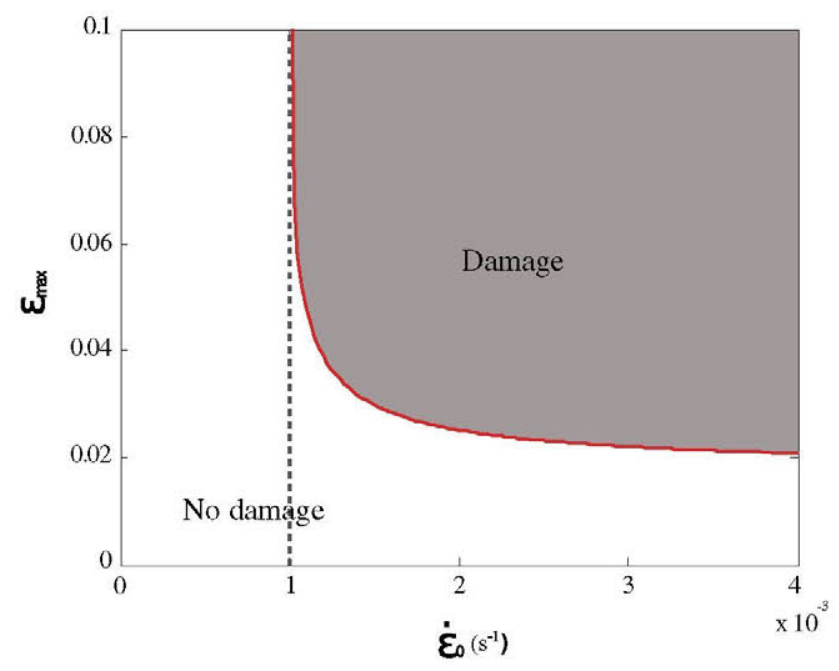

Fig. 2 Damage space of the analytical model: the vertical dashed line corresponds to $\dot{\varepsilon}_{0}=\frac{\sigma_{0}}{\eta_{1}^{+}}$and the red boundary line to the equation $\varepsilon_{\max }=-\tau^{+} \dot{\varepsilon}_{0} \ln \left(1-\frac{\sigma_{0}}{\eta_{1}^{+} \dot{\varepsilon}_{0}}\right)$ where $\tau^{+}=\eta_{1}^{+} / E$ where $\tau^{-}=\eta_{\mathrm{eq}} / E$ and $\eta_{\mathrm{eq}}=\left(1 / \eta_{1}^{-}+1 / \eta_{2}^{-}\right)^{-1}$. In these expressions, $\varepsilon_{\mathrm{m}, \mathrm{a}}^{*}$ is the microscopic axial strain at the onset of unloading, and $\varepsilon_{\mathrm{D}}^{*}$ is the corresponding accumulated damage strain. Two cases are then possible:

- The undamaged case (white region in Fig. 2):

$$
\left\{\begin{array}{l}
\varepsilon_{\mathrm{m}, \mathrm{a}}^{*}=\tau^{+} \dot{\varepsilon}_{0}\left(1-e^{-\frac{\varepsilon_{\mathrm{max}}}{\varepsilon_{0} \tau^{+}}}\right) \\
\varepsilon_{\mathrm{D}, \mathrm{a}}^{*}=0
\end{array}\right.
$$

where $\tau^{+}=\eta_{1}^{+} / E$.

- The damaged case (gray region in Fig. 2):

$$
\left\{\begin{array}{l}
\varepsilon_{\mathrm{m}, \mathrm{a}}^{*}=\left[\Sigma(1+\alpha)-\tau^{*} \dot{\varepsilon}_{0}\right] e^{-\frac{\frac{\varepsilon_{\max }-t_{0}^{\dagger}}{\varepsilon_{0}}}{\tau^{*}}}+\left(\tau^{*} \dot{\varepsilon}_{0}-\alpha \Sigma\right) \\
\varepsilon_{\mathrm{D}, \mathrm{a}}^{*}=\frac{\alpha}{1+\alpha}\left(\varepsilon_{\mathrm{m}, \mathrm{a}}^{*}-\Sigma\right)
\end{array}\right.
$$

where $\Sigma=\sigma_{0} / E, \alpha=E / k, \tau^{*}=(1+\alpha) \tau^{+}$and where

$$
t^{\dagger}=-\tau^{+} \ln \left(1-\frac{\Sigma}{\tau+\dot{\varepsilon}_{0}}\right)
$$

is the time at which damage first occurs when taking the onset of loading as the time reference.

Finally, in a configuration where the axon is clamped during stretch, the measurement of the output signal is made at a distance $\left(1+\varepsilon_{\mathrm{a}}\right) L$ from the input signal, where $L$ is the original axonal length. Because of the presence of tissue active molecular reorganization viscosity $\eta_{1}^{ \pm}$, the axon is able to grow. As such, the 1D discretized axon used for the simulation should be taken sufficiently long to account for this effect. The macroscopic axial strain $\varepsilon_{\mathrm{a}}(t)$ can be inferred from the microscopic one by noting that during the unloading:

$\dot{\varepsilon}_{\mathrm{a}}=\kappa \dot{\varepsilon}_{\mathrm{m}, \mathrm{a}}$

where $\kappa=\frac{\eta_{1}^{-}}{\eta_{1}^{-}+\eta_{2}^{-}}$.

The mechanical model thus depends on five parameters: $\tau^{+}$(the time constant of the tensile axonal active molecular reorganization), $\tau^{-}$(the equivalent time constant of the coupled stress-free axonal active molecular reorganization and viscosity of the surrounding structures), $\Sigma$ (the normalized damage threshold stress), $\alpha$ (a parameter characterizing the membrane damage evolution), and $\kappa$ (the proportion of macroscopic over microscopic strain rates during relaxation). It must be emphasized that these parameters can a priori be measured experimentally at the macroscopic tissue scale. The sixth and seventh parameters, $\gamma$ (the exponent representing the sensitivity of the damage model to small versus 
large deformation), and $\tilde{\varepsilon}$ (the microscopic membrane strain threshold at which the ion channels lose their potential) are coupling them to the electrophysiological model, see Eqs. $(9-10)$.

Unfortunately, experimental loadings such as the ones simulated in the following section cannot generally be performed at constant strain rates. In such cases, $\varepsilon_{\mathrm{m}, \mathrm{a}}^{*}$ and $\varepsilon_{\mathrm{D}}^{*}$ are simply determined by numerical integration of the system of equations (3) for the chosen loading, i.e., here, by numerically incrementally applying the experimentally observed macroscopic strain and calculating both $\varepsilon_{\mathrm{m}, \mathrm{a}}$ and $\varepsilon_{\mathrm{D}}$ at each increment. The resulting $\varepsilon_{\mathrm{m}, \mathrm{a}}^{*}$ and $\varepsilon_{\mathrm{D}}^{*}$ are then used in $\mathrm{Eq}$. (14) for the unloading (free of stress). Such numerical integration is done in the following section.

\subsection{Calibration}

The resulting model is finally calibrated according to the experimental results of tensile tests of guinea pig spinal cord white matter of Shi and Whitebone (2006). It must first be emphasized that the model proposed here provides the electrophysiological signal propagation within one unique axon, i.e., one AP, whereas the experimental results used for calibration and validation involve CAPs from a section of spinal cord (Shi and Whitebone 2006). As such the approximation is made that the simulated APs, deterministically determined, can be calibrated against the experimental CAPs (see also Sect. 4 below). To this end, $L \approx 10 \mathrm{~mm}$ of myelinated guinea pig axon was discretized in our in-house dedicated finite difference software Neurite. The electrophysiological parameters for guinea pig spinal cord axons were taken from literature when possible, and estimated from other animal and/or organs when no data was available (see Supplementary Material B).

Shi and Whitebone (2006) provide a measurement of the reduction in the CAP amplitude immediately after the tensile test and continuously for the subsequent $30 \mathrm{~min}$. In these experiments, the white matter strip is subjected to a set of slow $\left(\dot{\varepsilon}_{0}=0.006-0.008 \mathrm{~s}^{-1}\right)$ and fast maximum axial strain rates $\left(\dot{\varepsilon}_{0}=355-519 \mathrm{~s}^{-1}\right)$ for mild $\left(\varepsilon_{\max }=25 \%\right)$, moderate $\left(\varepsilon_{\max }=50 \%\right)$ and severe $\left(\varepsilon_{\max }=100 \%\right)$ axial strains loads. The authors recorded the exact evolution of the strain rate for each loading case, and the same loading was applied numerically to the set of equations (3) for all three cases.

Despite observations of stress concentrations at the NRs during stretch injury, the relation between axonal, myelin and glial stiffnesses, as well as the role of oligodendrocytes and paranodal axo-glial adhesions in the "glueing" of all three components remain largely controversial (Shreiber et al. 2009). As a consequence and in the absence of more conclusive evidences, the case where all the strain is sustained equally by the NRs and the IRs is considered here (i.e., $v=\chi$ ). The methodology for the model calibration is provided in Supplementary Material B, with the final set of parameters in Tables 3,4, and 5, and the final results for a triggering current of $0.04 \mathrm{nA}$ during $3 \mathrm{~ms}$ are shown in Fig. 3 . It is finally important to emphasize the fact that effective values of the resistivity and electric constant of the membrane or myelin layers are used here. The non-effective counterparts also used in other formulations can be recovered by dividing or multiplying the effective values by the thickness of the membrane or myelin layers. See Koch (1999) for more details. The new resting potential was evaluated by taking the minimum of the potential in the measured point for the last ms of each simulation and each AP was measured with respect to this value. A noticeable change in the resting potential was observed in the slow-severe and fast-mild cases. Additionally, for these cases (and not in the other cases), new (one stable and the other one non-stable) equilibriums were observed at $30 \mathrm{~min}$ by which, once triggered by the input signal, the first output signal is followed by a train of other peaks. Subsequent signals are then much more attenuated that the first one (not shown). In the proposed work, only the first destabilizing peak was considered for the calibration, but further studies will be needed to classify such behavior, as it seems to indicate not only two (Volman and $\mathrm{Ng} 2013$ ) but three regimes (stable-unstable-stable when increasing the damage).

A time step of $32.9868 \mathrm{~ns}$ (calculated as a factor 0.8 of the critical time step) and element sizes of $80 \mu \mathrm{m}$ for the IRs and $2.1 \mu \mathrm{m}$ for the NRs were used for the reference simulation. The deformed elements were then elongated according
Table 3 Geometrical model parameters

\begin{tabular}{lll}
\hline Parameter & Value & Reference \\
\hline Membrane reference thickness $\left(h_{0}\right)$ & $4 \mathrm{~nm}$ & Koch (1999) \\
Axon reference diameter $\left(d_{0}\right)$ & $3 \mu \mathrm{m}$ & Ouyang et al. (2010) \\
Number of myelin layers in the IRs $\left(n_{\mathrm{my}}\right)$ & $\approx 45$ & Hildebrand and Hahn (1978) \\
Myelin layer reference thickness $\left(h_{\mathrm{my}, 0}\right)$ & $\approx 18 \mathrm{~nm}$ & Jensen et al. (2011) \\
Length of nodes of Ranvier & $2.1 \mu \mathrm{m}$ & Ouyang et al. (2010) \\
Internodal distance & $\approx 800 \mu \mathrm{m}$ & Jacobs and Cavanagh (1969), Ibrahim et al. \\
& & $(1995)$, Koch (1999) \\
\hline
\end{tabular}


Table 4 Electrical model parameters; note that effective values are used (non-effective values can be recovered by multiplication or division by the membrane/myelin layer thickness; see Koch 1999)

\begin{tabular}{|c|c|c|}
\hline Parameter & Value & Reference \\
\hline Axial cytoplasm resistivity $\left(\rho_{\mathrm{a}}\right)$ & $1.87 \Omega \mathrm{m}$ & Koch (1999) \\
\hline Membrane resistivity $\left(\rho_{\mathrm{m}}\right)$ & $2.5 \times 10^{9} \Omega \mathrm{m}$ & Koch (1999) \\
\hline Myelin layer resistivity $\left(\rho_{\mathrm{my}}\right)$ & $4.44 \times 10^{6} \Omega \mathrm{m}$ & Chomiak and $\mathrm{Hu}(2009)$ \\
\hline Membrane electric constant $\left(C_{\mathrm{m}}\right)$ & $4 \times 10^{-11} \mathrm{~F} / \mathrm{m}$ & $\begin{array}{l}\text { Hodgkin and Huxley (1952), Koch } \\
\text { (1999), Chomiak and Hu (2009) }\end{array}$ \\
\hline Myelin layer electric constant $\left(C_{\mathrm{my}}\right)$ & $1.08 \times 10^{-10} \mathrm{~F} / \mathrm{m}$ & Chomiak and $\mathrm{Hu}(2009)$ \\
\hline Resting potential ( $\left.V_{\text {rest }}\right)$ & $-65.5 \mathrm{mV}$ & Boucher et al. (2012) \\
\hline $\mathrm{Na}_{\mathrm{v}}$ reversal potential $\left(E_{\mathrm{Na}, 0}\right)$ & $49.5 \mathrm{mV}$ & Hodgkin and Huxley (1952), Koch (1999) \\
\hline $\mathrm{K}_{\mathrm{v}}$ reversal potential $\left(E_{\mathrm{K}, 0}\right)$ & $-77.5 \mathrm{mV}$ & Hodgkin and Huxley (1952), Koch (1999) \\
\hline Leak conductivity $\left(G_{\mathrm{L}}\right)$ & $1.2 \times 10^{-8} \mathrm{~S} / \mathrm{m}$ & Hodgkin and Huxley (1952), Koch (1999) \\
\hline $\mathrm{Na}_{\mathrm{v}}$ reference conductivity $\left(\bar{G}_{\mathrm{Na}}\right)$ & $4.8 \times 10^{-6} \mathrm{~S} / \mathrm{m}$ & Hodgkin and Huxley (1952), Koch (1999) \\
\hline $\mathrm{K}_{\mathrm{v}}$ reference conductivity $\left(\bar{G}_{\mathrm{K}}\right)$ & $1.44 \times 10^{-6} \mathrm{~S} / \mathrm{m}$ & Hodgkin and Huxley (1952), Koch (1999) \\
\hline
\end{tabular}

Table 5 Calibrated mechanical model parameters

\begin{tabular}{ll}
\hline Parameter & Value \\
\hline$\tau^{+}$ & $18.08 \mathrm{~s}$ \\
$\Sigma$ & $1.8 \times 10^{-2}$ \\
$\alpha$ & 0.9 \\
$\tau^{-}$ & $111.5 \mathrm{~s}$ \\
$\kappa$ & 0.5 \\
$\gamma$ & 2 \\
$\tilde{\varepsilon}$ & 0.1 \\
\hline
\end{tabular}

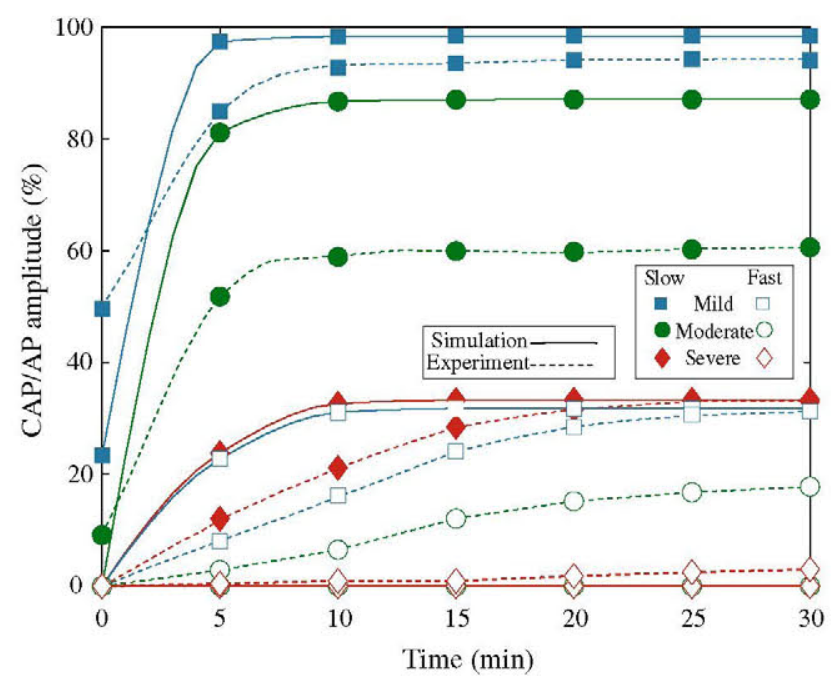

Fig. 3 CAP (experiment) and AP (simulation) amplitude evolution during unloading in percentage of the reference one (no strain): the experimental results from Shi and Whitebone (2006) (dashed lines), and our simulation results (solid lines) for slow and fast strain rate and for mild $(25 \%)$, moderate $(50 \%)$ and severe $(100 \%)$ maximum macroscopic axial tensile strain

to the microscopic strain but did not need to be refined (convergence was still observed). The time step was recalculated accordingly.
It must be emphasized that the experimental work of Shi and Whitebone (2006) was used for calibration for its meticulous and complete analysis of CAP evolutions over a wide range of strain and strain rate. However, the model can easily be calibrated against any other system, providing that enough data points allow for the identification of all model parameters.

\section{Discussion}

\subsection{Irreversible damage thresholds}

A first observation is that the model naturally provides a set of thresholds for tissue/cell irreversible damage, see Fig. 2. Indeed, below a given threshold of $\sigma_{0} / \eta_{1}^{+}$, a constant strain rate $\dot{\varepsilon}_{0}$ applied to the tissue is not overcoming its dynamic active accommodation (e.g., membrane/tissue turnover, (de)polymerization and (de)lipidation), and the membrane molecular bonds thus remain undamaged, independently of the applied strain. However, above this strain rate threshold, a strain threshold exists at

$\varepsilon_{\max }=-\tau^{+} \dot{\varepsilon}_{0} \ln \left(1-\frac{\sigma_{0}}{\eta_{1}^{+} \dot{\varepsilon}_{0}}\right)$

above which irreversible microscopic damage eventually occurs. Any tensile test characterized by a $\left(\varepsilon_{\max }, \dot{\varepsilon}_{0}\right)$ pair situated in the gray area of Fig. 2 will imply that a component of the AP amplitude decrease will never be recovered (as observed in all cases in Fig. 3). Accordingly, any pair situated in the white area will guarantee full recovery of the electrophysiological functions after sufficient time has elapsed. Such behavior is in line with experimental results analyzing axonal growth under stretch on the one hand (Dennerll et al. 1989), and increased damage for increased strains and strain rates on the other hand (Shi and Whitebone 2006). 
The proposed model also suggests that axons populated by cardiac $\mathrm{Na}_{\mathrm{v}}$ isotype $\left(\mathrm{Na}_{\mathrm{v}} 1.5\right)$, observed to provide a reversible behavior after stretch (Wang et al. 2009) would simply be characterized by a higher $\sigma_{0} / \eta_{1}^{+}$factor and/or $\varepsilon_{\max }$ than the ones populated by the $\mathrm{Na}_{\mathrm{v}} 1.4$ and $\mathrm{Na}_{\mathrm{v}} 1.6$ "irreversible" isotypes, thus remaining in the non-damaged area of Fig. 2. The $\mathrm{Na}_{\mathrm{v}} 1.4$ and $\mathrm{Na}_{\mathrm{v}} 1.6$ isotypes, on the contrary, would thus present a higher susceptibility to irreversible damage. The presence of the underlying cortical axoplasm and its intrinsic mechanical relation to the ion channel most likely conditions these parameters.

In addition to the electrophysiological data being routinely identified for all ion channels, we propose here the systematical identification of the parameters of Eq. (19) for each one of the ion channels. This will eventually participate in categorizing not only the physiological properties of the ion channels, but also their propension at sustaining or not irreversible damage.

\subsection{Model analysis}

The results of Fig. 3 confirm the ability of the model to reproduce the main features of the electrophysiological properties during unloading of a damaging injury. A discrepancy with the experimental results emerges for the slow case at moderate strain and for fast loading cases at moderate and severe tensile strains. For the slow case, a strong instability leading to a sudden decrease in AP was observed when lowering further. Note that this sudden "switch" is actually happening for the slow-severe and fast-mild cases and seems to be automatically accompanied by a change in the equilibrium potential to $\sim-55 \mathrm{mV}$, consistent with the simulations of Boucher et al. (2012) for a left-shift of $20 \mathrm{mV}$. The simulation results are thus not incompatible with the experimental ones as the experimental CAPs are actually an average of multiple APs, for which an upper bound is simulated here. Future work on the averaging of simulated APs should tackle this issue. The fast case discrepancy is also not surprising considering the simple mechanical model proposed here: the overevaluation of the damage should be corrected with a more adequate extracellular matrix model. Current work is focusing on such extension.

As discussed earlier, the overall behavior of the model potential propagation might be driven by the leaking behavior of $\mathrm{Na}_{\mathrm{V}}$ channels, in agreement with experimental campaigns aimed at enhancing axonal conduction after SCIs by $\mathrm{Na}_{\mathrm{v}}$ channel blockade (Hains et al. 2004). Our model exhibits two different alteration mechanisms. The first one is exclusively due to geometrical effects: the elongation of the axons at constant volume increases the membrane surface available to ion and electron exchanges with the extra-cellular medium, see Eq. (5). The incompressibility approximation is motivated by the assumption that minor swelling and fluid fluxes eventu- ally leading to significant mass variations would only occur on larger time scales. This leads to a faster decrease in the potential, and eventually a sudden collapse of the signal if the signal reaching the $\mathrm{Na}_{\mathrm{v}}$ channels is not strong enough to activate them. This mechanism alone was tested with the model proposed here and was shown to be insufficient to decrease the signals as much as observed experimentally by (Shi and Whitebone 2006). The second mechanism involves an additional damage-based ion channel properties alteration, see Eqs. (9-10). It must be emphasized that this alteration solely depends on $\varepsilon_{\mathrm{m}}$ and can thus be reversible or not depending on the mechanical behavior; see Eq. (19). Figure 4 shows the simulated potentials (a) and ion channel (first and twelfth NRs) currents (b), for (i) the reference case, and slow-severe case (at $t=30 \mathrm{~min}$ ) with (ii) only with geometrical alteration (no ion channel damage alteration), (iii) without ion channel damage alteration for $K_{v}$, and (iv) the full model. The effect of geometrical alteration on the potential is mainly measured in terms of delay of the signal (whether potential in Fig. 4a or current in Fig. 4b); this is, however, not due to the stretch-induced elongation (as seen in the 12th NR of Fig. 4b, where damaged signal and reference signal arrival times remain relatively close) but to the active growth of the axon (as seen in the arrival of the potential at the point of measurement). A video of the propagation of the potential versus microscopically deformed length is provided as Supplementary Material $\mathrm{C}$ and shows that the damaged signal indeed travels at the same speed with respect to the microscopically deformed referential. However, the active growth of the axon due to the presence of tissue active molecular reorganization viscosity $\eta_{1}^{ \pm}$implies a point of measurement in this referential at a further distance (both points of measurement are indicated by arrows in the video), thus confirming the delay of signal observed experimentally (Shi and Pryor 2002).

The model predicts a similar left-shift (see 1st NR of Fig. 4b) as reported by Wang et al. (2009) but does not seem to exhibit the immediate post-traumatic larger $\mathrm{Na}_{\mathrm{v}}$ currents reported in the same reference. However, the patch suction loading used in this reference implies a very localized damage mostly characterized by blebbing, i.e., strained membrane with underlying cortical axoplasm disruption. Figure 4 shows that such type of "leak" can be obtained by only accounting for geometrical alteration, i.e., without Eqs. (910). This could be explained by the fact that suction involves a very localized and severe membrane bending, possibly straining and damaging the membrane without directly damaging the ion channels. Additionally, a large immediate increase in $\mathrm{Na}_{\mathrm{v}}$ current implies a priori a stronger potential and faster signal, which is in contradiction with most of the experimental campaigns making use of stretching (Shi and Pryor 2002; Shi and Whitebone 2006) or blast loading (Connell et al. 2011). However, the model exhibits different equilib- 
Fig. 4 Simulated potentials (a) and ion channel (first and twelfth NRs) currents (b), for (i) the reference case, and slow-severe case (at $t=30 \mathrm{~min}$ ) with (ii) the full model, (iii) only with geometrical alteration (no damage alteration), and (iv) without damage alteration for $\mathrm{K}_{\mathrm{v}}$
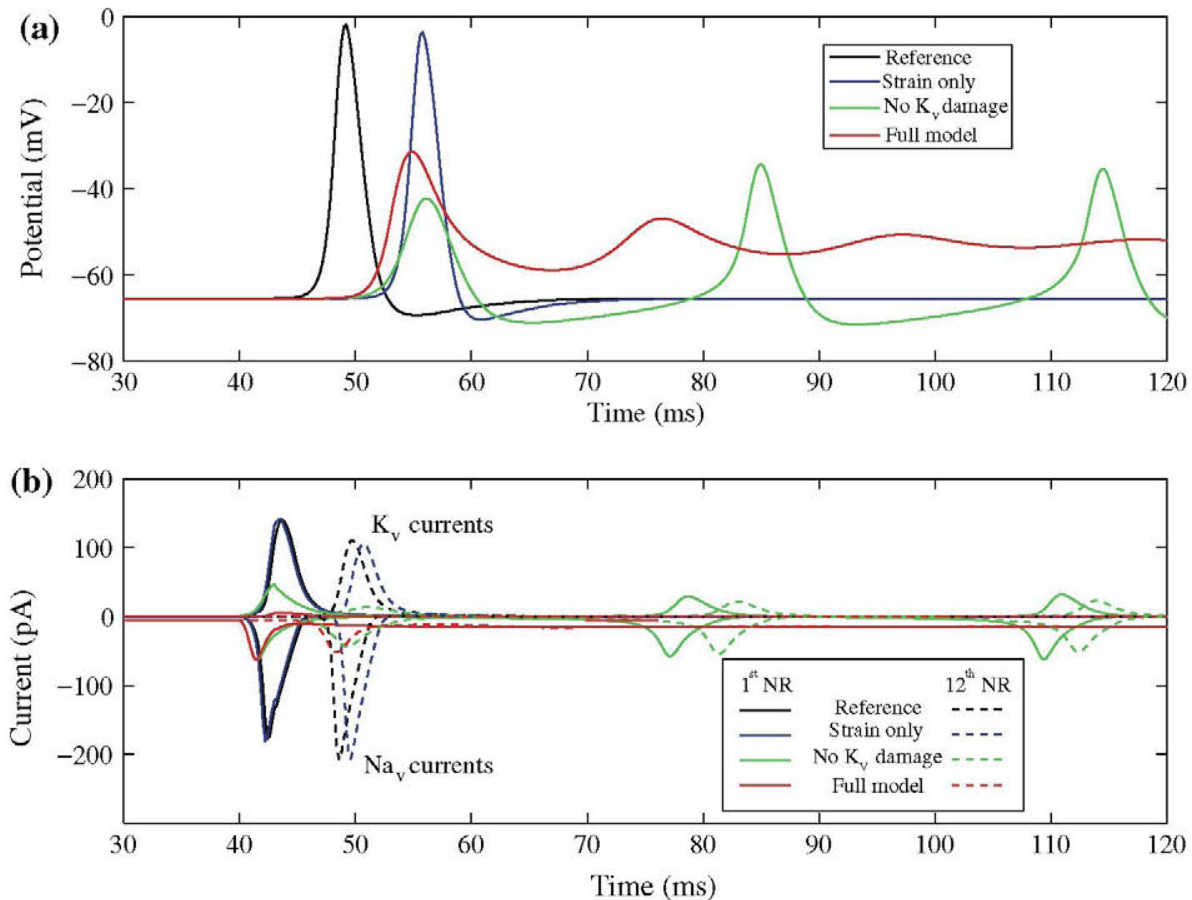

Table 6 Equilibrium ion channel currents

\begin{tabular}{llc}
\hline Case & $\mathrm{Na}_{\mathrm{v}}(\mathrm{pA})$ & $\mathrm{K}_{\mathrm{v}}(\mathrm{pA})$ \\
\hline Reference & -0.24 & 0.87 \\
Slow-mild & -0.51 & 0.23 \\
Slow-moderate & -1.6 & 0.0012 \\
Slow-severe & -5.52 & -0.0019 \\
Fast-mild & -5.09 & -0.0024 \\
Fast-moderate & -7.91 & 0 \\
Fast-severe & -7.91 & 0 \\
\hline
\end{tabular}

rium (i.e., without triggering signal) currents, see Table 6. These values are orders of magnitude lower than on Fig. 4 during AP propagation but the $\mathrm{Na}_{\mathrm{v}}$ equilibrium (without AP) current is drastically increased between the reference cases and the damaged cases. This would indicate that $\mathrm{Na}_{\mathrm{v}}$ leaks observed post-trauma are either a slower mechanism occurring at a later stage (Hains et al. 2004) - see also discussion below on damage time scales - or actually a more discrete phenomenon linked to a different equilibrium involving a much larger ionic current without any $\mathrm{AP}$ propagation.

Finally, the simulations in Fig. 4 exhibited an important influence of the damage-based $\mathrm{K}_{\mathrm{V}}$ alteration on the $\mathrm{Na}_{\mathrm{v}}$ current and AP. This influence is particularly marked in terms of stability of the AP and change in resting potential. Additionally, the model is assuming that the parameters $\gamma$ and $\tilde{\varepsilon}$ in Eqs. (9) and (10) are the same for both $\mathrm{Na}_{\mathrm{v}}$ and $\mathrm{K}_{\mathrm{v}}$, thus indicating a need for a potential differentiated calibration of both sets of parameters. Additionally a much lower equilib- rium current than in the reference case is observed for the damaged cases in Table 6 , indicating that the damage-based leaks (if indeed mainly caused by this equilibrium currents alterations) are mostly affecting $\mathrm{Na}_{\mathrm{v}}$. This observation suggests that the modeling choice of altering $\mathrm{K}_{\mathrm{v}}$ along with $\mathrm{Na}_{\mathrm{v}}$ should have significant influence on the overall stability of the system but that the experimentally observed extracellular $\mathrm{K}^{+}$ longer term accumulation in post-traumatic rat hippocampal slices might indeed be due to glial activity and not neuronal leak (D'Ambrosio et al. 1999).

\subsection{Parameters analysis}

It must be emphasized that other families of solutions can be found for other more realistic values of $v$ accounting for the cross-links of the axon overlaps in the white matter. However, the purpose of this article being mainly to introduce the model, the assumption of homogeneous deformation can be considered as a first satisfactory approximation for this tensile case. Additional experimental methods can actually participate in identifying the value of $v$. For instance, in the compression experiments of Ouyang et al. (2010), coherent anti-Stokes Raman spectroscopy and immunohistochemistry identified that the ratio between the length and diameter of the NRs increased by a factor of four between rest and onset of unloading. Discarding a possible demyelination and assuming one more time the incompressibility of the NRs, the coefficient $v$ can thus be estimated directly from the macroscopic strains for this loading condition. Further experimental work should systematically involve such mea- 
surement to help identifying the value of $v$ [note that paranodal demyelination consideration coupled with appropriate ion channels density observation (Ichimura et al. 2005) could also be factored in in future models]. A complete parameter study is provided in Supplementary Material B for the remaining parameters.

Finally, it must be emphasized that the Hodgkin-Huxley model and its corresponding parameters are certainly not fully adequate for the guinea pig spinal cord. However, Fig. 3 exhibits a relatively good fit. Additionally, this work is aiming at providing a new framework for which mechanical and electrophysiological models can easily be changed for other more adequate models.

\subsection{Membrane integrity}

The use of a microscopic membrane strain $\varepsilon_{\mathrm{m}}$ naturally defines a framework for a measure of membrane integrity. Shi and Whitebone (2006) assessed it by use of horseradish peroxidase (HRP) exclusion tests. In this reference, the density of axons labeled with HRP (axons $/ \mathrm{mm}^{2}$ ) was calculated for each case of Fig. 3 at $t=0 \mathrm{~min}$ and $t=30 \mathrm{~min}$. Assuming that the microscopic membrane strain is directly proportional to the leak of HRP in one axon, we propose to corroborate the hypothesis that $\varepsilon_{\mathrm{m}}$ can be used as a measure for membrane integrity by plotting the density of axons labeled with HRP (but divided by $\left(1+\varepsilon_{\mathrm{a}}\right)$ to account for the volume-conserving cross-section reduction during stretch). The results are shown in Fig. 5. A linear tendency can be seen, confirming the ability of $\varepsilon_{\mathrm{m}}$ at capturing membrane integrity. Note that the two points at zero density were not taken into account for the lin-

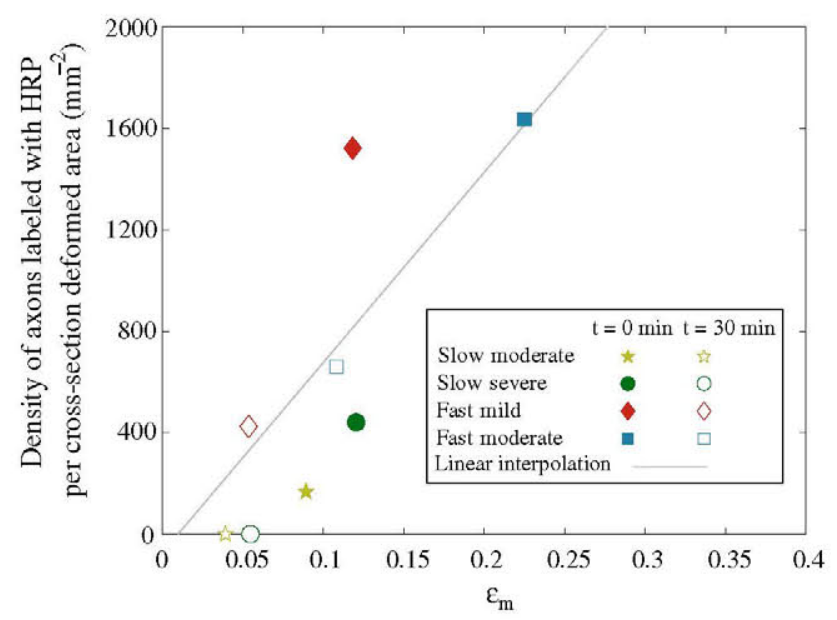

Fig. 5 Density of axons labeled with HRP per cross-section deformed area (Shi and Whitebone 2006) versus corresponding model microscopic membrane strain $\varepsilon_{\mathrm{m}}$, and linear regression; the points corresponding to zero density were discarded from the linear regression as a membrane disruption smaller than the size of HRP $(5 \mathrm{~nm})$ cannot be detected (Shi and Whitebone 2006) ear regression as the membrane holes that allow the passage of HRP are estimated to be $\sim 5 \mathrm{~nm}$ large, which implies that disruption smaller than the size of HRP will go undetected by HRP (Shi and Whitebone 2006).

\subsection{Damage time scales}

A similar study focused on long-term (up to 14 days) electrophysiological dysfunctions after blast trauma has highlighted the need for the consideration of another time scale (Park et al. 2013). More specifically, whereas the model proposed here accounts for two alteration mechanisms-namely, geometrical deformation and immediate ion channel damagethis experimental work highlights the role of neurofilament expression alteration. After an injury, neurofilaments have been observed to accumulate in the soma, suggesting an alteration of the transport mechanisms in axons and thus changes in axonal caliber (Park et al. 2013). As demonstrated by the model proposed here, geometrical deformation of the axon invariably leads to changes in the signal propagation. Such expression alteration will then further decrease the ability of the axon to propagate an electrical signal on a longer-term basis (days).

Additionally, axonal injury has been shown to lead to cleavage of $\alpha$ II-spectrin (a cortical axoplasm protein), either attacked by $\mathrm{Ca}^{2+}$ proteases, originally overactivated by $\mathrm{Na}^{+} / \mathrm{Ca}^{2+}$ exchangers (Wang et al. 2009) overwhelmed by a possible initial $\mathrm{Na}_{\mathrm{v}}$ leak, or simply mechanically sheared (Park et al. 2013). This cleavage then reduces the association of this protein with ankyrin $\mathrm{G}$ (a protein involved in the anchoring of $\mathrm{Na}_{\mathrm{v}}$ channels), thus leading to a diffusion and reorganization of $\mathrm{Na}_{\mathrm{v}}$ channels at the NRs (Park et al. 2013). Again, such change is suggested to lead to a decrease in AP on a long-term basis.

In addition to the geometrical and damage alterations highlighted above, both observations point toward a third alteration mechanism due to longer-term mechanisms such as diffusion ( $\mathrm{Na}_{\mathrm{v}}$ in the membrane) or active transport alteration (neurofilaments in the axoplasm). The model proposed here is built on the same code as the one proposed by García et al. (2012) for the simulation of axonal growth based on diffusion and active transport of proteins. Future work could focus on the coupling of the diffusive and active transport mechanisms of the latter model with the one proposed here. This should then allow for the consideration of the longer-term mechanisms discussed here.

Other possible mechanisms could involve a slower timedependent recalibration of $E_{\mathrm{L}}$ through Eq. (8) as the ion homeostasis exchangers try to reaccommodate the changes in potentials due to $\mathrm{Na}_{\mathrm{v}}$ and $\mathrm{K}_{\mathrm{v}}$ after damage to rebalance the potential to its resting potential. 


\subsection{Future work}

In addition to the longer-term mechanism discussed above, the natural extensions of this model are pointing toward a three-dimensionalization of the framework. This will involve the adoption of less restrictive solvers such as the finite element method. Such extension is needed for the identification of more complex loading states such as local compression, blast, inertia, or more generally loadings involving a stress gradient within the thickness of the axon. Even though the model proposed here should a priori behave appropriately under large macroscopic deformation because only the microscopic deformation (a fraction of the former) is concerned by the small strain elastic law, a large deformation framework should enhance the proposed formulation considerably and will be proposed along with the finite element formulation.

The observation of strong instabilities for different level of damage and different parameters (especially taking into account, or not, $\mathrm{K}_{\mathrm{V}}$ alterations), as well as the fact that once a new resting potential has been reached, subsequent APs seem to behave differently (in general much more attenuated) will be tackled in future work. This includes aspects of AP propagation, such as a deeper study of unstable saltatory behaviors in damaged myelinated axons (Boucher et al. 2012) or in demyelinated axon (Waxman and Brill 1978; Coggan et al. 2010) and their relation to mechanical loading.

Finally, as highlighted earlier, the model proposed here provides the AP propagation within one unique axon. The experimental results used for calibration and validation involve CAPs. As such, a more genuine comparison should be done by averaging (based on the corresponding experimental methods used for the measurement) the behavior of many axons using random statistics related to their diameters, the exact positions of the NRs or the lengths of the IRs. Future work will tackle this effort.

\section{Conclusion}

We propose here a new multiscale framework coupling mechanical and electrophysiological properties. To the best of the knowledge of the authors and as suggested by a recent review (Gupta and Przekwas 2013), this model is the first of the kind. It is constituted of four main building blocks whose parameters have been taken from actual experiments, leaving only seven remaining free parameters. These parameters are not only representative of purely mechanical propertiesmeasurable experimentally at the macroscopic level-but also of the molecular reorganization or damage that would be expected in living tissue under traumatic stress. The model was eventually thoroughly utilized to analyze recent works on ion channel leak and membrane integrity, among others, and the different time scales possibly involved in the corresponding mechanical damage were discussed. As a conclusion, this model paves the road to a new series of models able to predict the alteration of neuronal electrophysiological functions under external damaging load (both reversible and irreversible), thus linking mechanical injuries to subsequent acute functional deficits. By directly linking macroscale mechanical loading, microscale deformation, molecular reorganization and functional deficits, such model ultimately participates to the overarching goal of understanding the causes and consequences of spinal cord and traumatic brain injuries.

Acknowledgments The research leading to these results has received funding from the European Research Council under the European Union's Seventh Framework Programme (FP7 2007-2013)/ERC Grant Agreement No. 306587, from the Spanish Ministry of Science (TIN2010-21289-C02-02), and the Cajal Blue Brain Project, the Spanish partner of the Blue Brain Project. The authors gratefully acknowledge the computer resources, technical expertise and assistance provided by the Supercomputing and Visualization Center of Madrid (CeSViMa).

\section{References}

Alford P, Dabiri B, Goss J, Hemphill M, Brigham M, Parker K (2011) Blast-induced phenotypic switching in cerebral vasospasm. Proc Natl Acad Sci 108:12705-12710

Babbs CF, Shi R (2013) Subtle paranodal injury slows impulse conduction in a mathematical model of myelinated axons. PLoS One 8(7):e67767

Boucher P-A, Joós B, Morris CE (2012) Coupled left-shift of $\mathrm{Na}_{v}$ channels: modeling the $\mathrm{Na}^{+}$-loading and dysfunctional excitability of damaged axons. J Comput Neurosci 33:301-319

Cernak I, Noble-Haeusslein L (2010) Traumatic brain injury: an overview of pathobiology with emphasis on military populations. J Cereb Blood Flow Metab 30:255-266

Chomiak T, Hu B (2009) What is the optimal value of the g-Ratio for myelinated fiber in the rate CNS? A theoretical approach. PLoS ONE 4:e7754

Cloots RJH (2011) Multi-scale mechanics of traumatic brain injury $\mathrm{PhD}$ thesis, Eindhoven University of Technology

Cloots RJH, van Dommelen JAW, Kleiven S, Geers MGD (2013) Multiscale mechanics of traumatic brain injury: predicting axonal strains from head loads. Biomech Model Mechanobiol 12:137-150

Connell S, Gao J, Chen J, Shi R (2011) Novel model to investigate blast injury in the central nervous system. J Neurotrauma 28:1229-1236

Coggan JS, Prescott SA, Bartol TM, Sejnowski TJ (2010) Imbalance of ionic conductances contributes to diverse symptoms of demyelination. Proc Natl Acad Sci 107(48):20602-20609

D'Ambrosio R, Maris DO, Grady MS, Winn HR, Janigro D (1999) Impaired $\mathrm{K}^{+}$homeostasis and altered electrophysiological properties of post-traumatic hippocampal glia. J Neurosci 19:8152-8162

Dennerll TJ, Lamoureux P, Buxbaum RE, Heidemann SR (1989) The cytomechanics of axonal elongation and retraction. J Cell Biol 109:3073-3083

García JA, Peña JM, McHugh S, Jérusalem A (2012) A model of the spatially dependent mechanical properties of the axon during its growth. Comput Model Eng Sci 87:411-432

Goldstein L, Fisher A, Tagge C, Zhang X-L, Velisek L, Sullivan J, Upreti C, Kracht J, Ericsson M, Wojnarowicz M, Goletiani C, Maglakelidze G, Casey N, Moncaster J, Minaeva O, Moir R, Nowinski C, Stern 
R, Cantu R, Geiling J, Blusztajn J, Wolozin B, Ikezu T, Stein T, Budson A, Kowall N, Chargin D, Sharon A, Saman S, Hall G, Moss W, Cleveland R, Tanzi E, Stanton P, McKee A (2012) Chronic traumatic encephalopathy in blast-exposed military veterans and a blast neurotrauma mouse model. Sci Transl Med 4:1-16

Gupta RK, Przekwas A (2013) Mathematical models of blast-induced TBI: current status, challenges, and prospects. Front Neurol 4(59):121

Hains BC, Saab CY, Lo AC, Waxman SG (2004) Sodium channel blockade with phenytoin protects spinal cord axons, enhances axonal conduction, and improves functional motor recovery after contusion SCI. Exp Neurol 188:365-377

Hicks R, Fertig S, Desrocher R, Koroshetz W, Pancrazio J (2010) Neurological effects of blast injury. J TRAUMA Inj Infect Crit Care 68:1257-1263

Hildebrand C, Hahn R (1978) Relation between myelin sheath thickness and axon size in spinal cord white matter of some vertebrate species. J Neurol Sci 38:421-434

Hodgkin A, Huxley A (1952) A quantitative description of membrane current and its application to conduction and excitation in nerve. $J$ Physiol 117:500-544

Ichimura H, Shiga T, Abe I, Hara Y, Terui N, Tsujino A, Ochiai N (2005) Distribution of sodium channels during nerve elongation in rat peripheral nerve. J Orthop Sci 10:214-220

Ibrahim M, Butt A, Berry M (1995) Relationship between myelin sheath diameter and internodal length in axons of the interior medullary velum of the adult rat. J Neurol Sci 133:119-127

Jacobs M, Cavanagh J (1969) Species differences in internode formation following two types of peripheral nerve injury. J Anat 105:295-306

Jensen T, Bech M, Bunk O, Menzel A, Bouchet A, Duc GL, Feidenhans'1 R, Pfeiffer F (2011) Molecular X-ray computed tomography of myelin in a rat brain. NeuroImage 57:124-129

Jérusalem A, Dao M (2012) Continuum modeling of neuronal cell under blast loading. Acta Biomaterialia 8:3360-3371

Koch C (1999) Biophysics of computation. Oxford University Press, Oxford

Markvin VS, Tanelian DL, Jersild RA Jr, Ochs S (1999) Biomechanics of stretch-induced beading. Biophys J 76:2852-2860

Moore D, Jérusalem A, Nyein M, Noels L, Jaffee M, Radovitzky R (2009) Computational biology-modeling of primary blast effects on the central nervous system. NeuroImage 47:T10-T20
Nyein M, Jason A, Yu L, Pita C, Joannopoulos J, Moore D, Radovitzky R (2010) In silico investigation of intracranial blast mitigation with relevance to military traumatic brain injury. Proc Natl Acad Sci 107:20703-20708

Ochs S, Jersild RA Jr, Pourmand R, Potter CG (1994) The beaded form of myelinated nerve fibers. Neuroscience 61:361-372

Ouyang H, Galle B, Li J, Nauman E, Shi R (2008) Biomechanics of spinal cord injury: a multimodal investigation using ex vivo guinea pig spinal cord white matter. J Neurotrauma 25:19-29

Ouyang H, Sun W, Fu Y, Li J, Cheng J, Nauman E, Shi R (2010) Compression induces acute demyelination and Potassium channel exposure in spinal cord. J Neurotrauma 27:1109-1120

Park E, Eisen R, Kinio A, Baker AJ (2013) Electrophysiological white matter dysfunction and association with neurobehavioral deficits following low-level primary bast trauma. Neurobiol Dis 52:150-159

Peter S, Mofrad M (2012) Computational modeling of axonal microtubule bundles under tension. Biophys J 102:749-757

Shi R, Pryor JD (2002) Pathological changes of isolated spinal cord axons in response to mechanical stretch. Neuroscience 110:765-777

Shi R, Whitebone J (2006) Conduction deficits and membrane disruption of spinal cord axons as a function of magnitude and rate of strain. J Neurophysiol 95:3384-3390

Shreiber D, Hao H, Elias R (2009) Probing the influence of myelin and glia on the tensile properties of the spinal cord. Biomech Model Mechanobiol 8:311-321

Volman V, Ng LJ (2013) Computer modeling of mild axonal injury: implications for axonal signal transmission. Neural Comput 25:136

Wang JA, Lin W, Morris T, Banderali U, Juranka PF, Morris CE (2009) Membrane trauma and $\mathrm{Na}^{+}$leak from $\mathrm{Na}_{v} 1.6$ channels. Am J Physiol Cell Physiol 297:C823-C834

Waxman SG, Brill MH (1978) Conduction through demyelinated plaques in multiple sclerosis: computer simulations of facilitation by short internodes. J Neurol Neurosurg Psychiatry 41:408-416

Wu L, Tjahjanto D, Becker G, Makradi A, Jérusalem A, Noels L (2013) A micro-meso-model of intra-laminar fracture in fiberreinforced composites based on a discontinuous Galerkin/cohesive zone method. Eng Fract Mech 104:162-183 